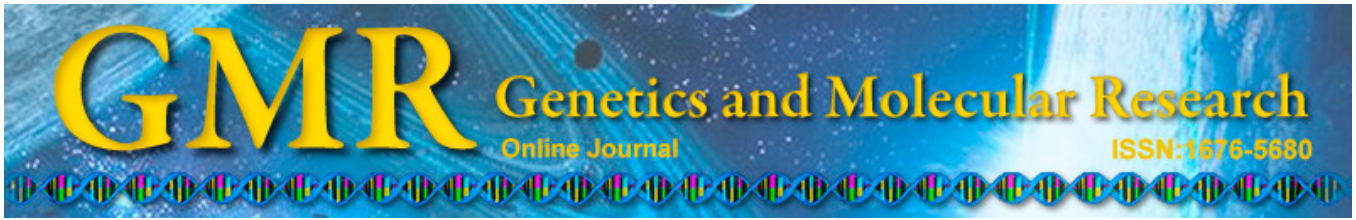

\title{
Acute toxic effects of sonodynamic therapy on hypertrophic scar fibroblasts of rabbit ears
}

\author{
W. Li ${ }^{1 *}$, J.F. Fei ${ }^{1 *}$, Q. Yang ${ }^{1}$, B.L. Li ${ }^{1}$, C. Lin ${ }^{1}$, Q. Yue ${ }^{2}$ and Q.G. Meng ${ }^{1}$ \\ ${ }^{1}$ Department of Study Center, The First Hospital of Harbin City, \\ Harbin Medical University, Harbin, China \\ ${ }^{2}$ Department of Study Center, Harbin Medical University, Harbin, China \\ *These authors contributed equally to this study. \\ Corresponding authors: Q. Yue / Q.G. Meng \\ E-mail: dlqiyue_qy@163.com/mengqigang_1@163.com
}

Genet. Mol. Res. 14 (2): 4203-4214 (2015)

Received September 8, 2014

Accepted December 12, 2014

Published April 28, 2015

DOI http://dx.doi.org/10.4238/2015.April.28.3

\begin{abstract}
The objective of this study was to observe the acute cytotoxic effects of hematoporphyrin monomethyl ether sonodynamic therapy (HMME-SDT) on hypertrophic scar fibroblasts of rabbit ears. We first assessed the effects of different irradiation times and HMME concentrations on the survival of hypertrophic scar fibroblasts using the 3-(4,5-dimethylthiazol-2-yl)-2,5-diphenyltetrazolium bromide (MTT) assay to determine the optimum irradiation time and HMME concentration. The hypertrophic scar fibroblast cell suspensions of the rabbit ears were divided into four groups, the survival rates were detected using the MTT assay, and the type of cell death was detected by Annexin V/propidium iodide (PI) double staining flow cytometry. Our results showed that HMME-SDT significantly reduced the viability of hypertrophic scar fibroblasts of rabbit ears at ultrasonic irradiation times of 30,60, and $90 \mathrm{~s}$, but not $10 \mathrm{~s}(\mathrm{P}<0.05)$. HMME alone had no significant effect on the cell survival rate at any irradiation time $(\mathrm{P}>$ $0.05)$. In contrast, the cell survival rate was significantly decreased at an irradiation time of $10 \mathrm{~s}$ and HMME concentrations of 20 and $50 \mu \mathrm{g} / \mathrm{mL}$ $(\mathrm{P}<0.05)$. Furthermore, Annexin V/PI double staining showed both
\end{abstract}


necrosis and apoptosis of the hypertrophic scar fibroblasts. Given our results, HMME might be an effective sound-sensitive agent for SDT as it has a significant lethal effect on hypertrophic scar fibroblasts of rabbit ear cultured in vitro. HMME-SDT may therefore provide a new method for the treatment of hypertrophic scar formation.

Key words: Sonodynamic therapy; Hypertrophic scar; Necrosis; Hematoporphyrin monomethyl ether; Apoptosis

\section{INTRODUCTION}

Sonodynamic therapy (SDT), also called sonodynamic chemistry therapy, consists of the intravenous injection of a specific dose of a sound-sensitive agent, or sonosensitizer, in patients followed by ultrasonic irradiation at a specific frequency and duration. This results in the accumulation of the sonosensitizer at the lesion site, which produces reactive oxygen molecules that attack and inhibit the growth of the diseased cells (Lv et al., 2012). The sonosensitizer and ultrasound are two important components of SDT. The discovery of novel sonosensitizers is an important focus of SDT research. Several sonosensitizer s are derived from photosensitizers, such as porphyrin complexes and porphyrin sources (e.g., hematoporphyrin, Photofrin, ATX-70, 5-ALA, quinolone, and HPD). Hematoporphyrin monomethyl ether (HMME) is a second-generation photosensitizer indigenously developed in China, which has the advantages of being a single component with stable performance, lower light-sensitivity, and an improved safety profile. However, so far no correlation studies have been conducted to determine its suitability as a sonosensitizer and its behavior in SDT (Dai et al., 2011). The current study investigated the sound sensitization and kinetic effects of HMME on hypertrophic scar fibroblasts of rabbit ear, which may provide a research model for the testing the feasibility of hypertrophic scar treatments.

\section{MATERIAL AND METHODS}

\section{Experimental animals}

Thirty-six rabbits with body weights ranging from 1.8 to $2.4 \mathrm{~kg}$ were purchased from the experimental animal center of Harbin Medical University, Harbin, China. The experiments were performed under license No. 2011-7812 and under clean conditions. HMME was developed by the Second Military Medical University, Harbin, China, with a specific concentration of $10 \mathrm{mg} / \mathrm{mL}$ and batch number 111209 . The rabbits were randomly divided into two groups: a normal control and a scar model group. The animals were acclimatized one week before the experiment at a temperature of $23 \pm 2^{\circ} \mathrm{C}$ and a relative humidity of $40 \pm 10 \%$. Water and solid feed was available ad libitum. Both groups were fed under the same conditions and environment throughout the experiment.

\section{Establishment of the rabbit ear hypertrophic scar model}

We established a rabbit ear hypertrophic scar model according to a modified version of a previously established method (Morris et al., 1997). The rabbits received anti-infective 
treatment after surgery and had access to food and water ad libitum. At day 21 post-surgery, the wound was found to be healing and was clearly protruding from the surface of the skin, which is indicative of hypertrophic scar formation.

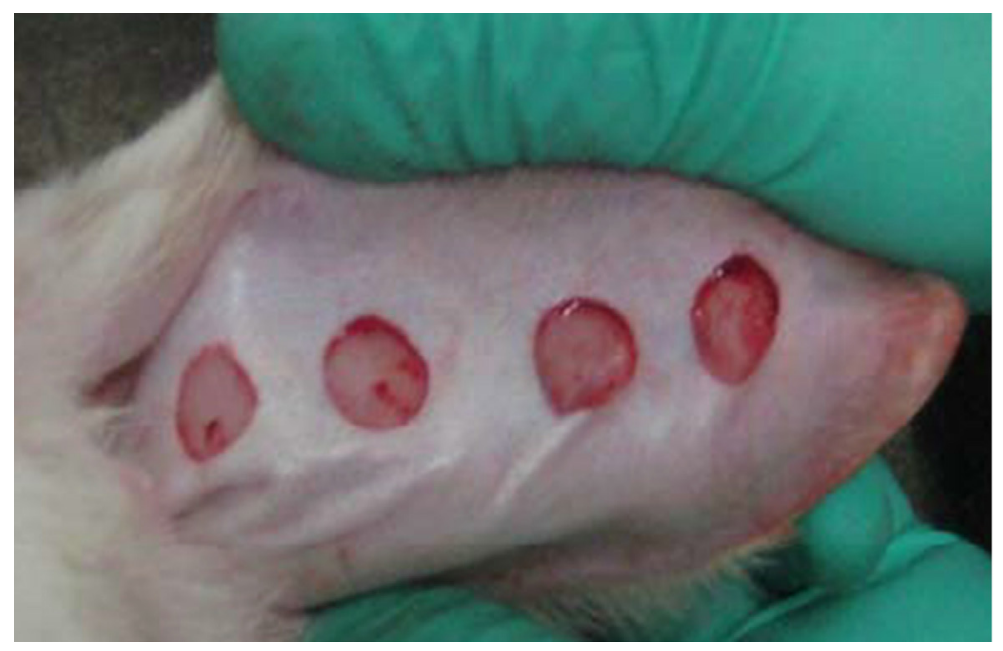

Figure 1. Molding of the hypertrophic scar

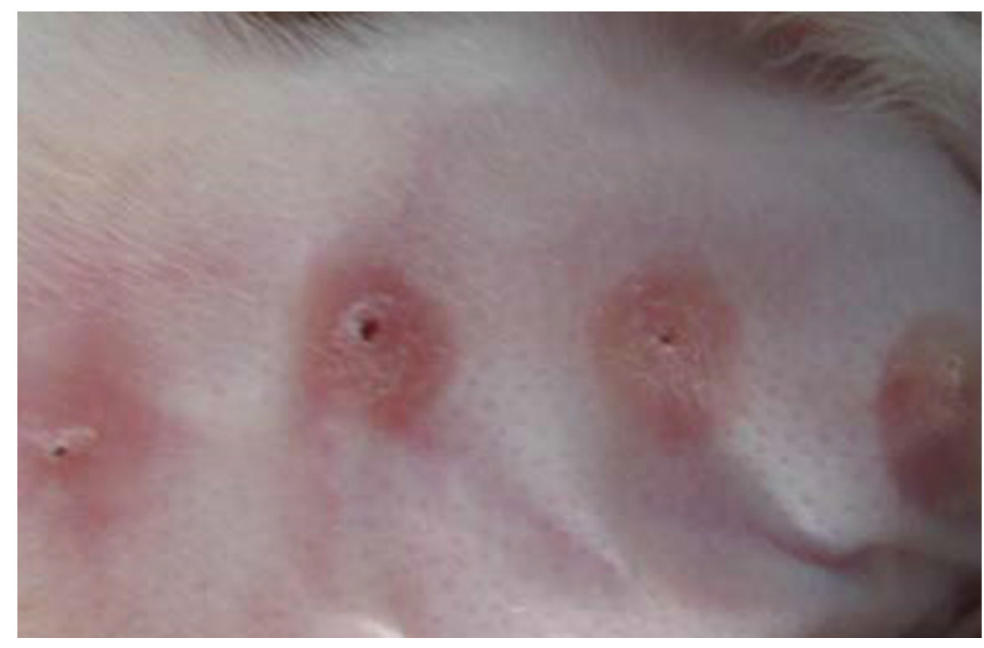

Figure 2. Hypertrophic scar model

\section{Cell recovery and cell growth}

After dissecting the hypertrophic scar tissue of the rabbit ears and recovering normal skin tissue cultured in vitro for $4 \mathrm{~h}$ the specimens were rinsed several times with NS. The epithelial tissue sections were subsequently removed, cut into $2 \mathrm{~mm} \times 2 \mathrm{~mm}$ pieces, placed in a sterile flask, and spread onto the flask's surface at a certain density by adding a small amount of medium to the flask. The tissue was cultured at the bottom of the flask and the cells were in 
the logarithmic growth phase after treatment and 3-4 subculture cycles. The fifth generation of cells was used in this study at which point the cell fusion was 70 to $80 \%$.

\section{Effects of HMME sonodynamic therapy on the survival of hypertrophic scar fibro- blasts of rabbit ears}

Cells that were in the logarithmic growth phase were digested and centrifuged, and a single cell suspension was prepared, adjusting the cell concentration to $5 \times 10^{5} / \mathrm{mL}$. All experiments were performed using $3 \mathrm{~mL}$ of this cell suspension. According to the results of previous experiments, the following experimental groups $(\mathrm{N}=3 \mathrm{~mL}$ cell suspensions) were used: a control, HMME, ultrasound alone, and acoustic power group. The ultrasonic irradiation conditions were $0.50 \mathrm{~W} / \mathrm{cm}^{2}$ for $10 \mathrm{~s}$ with 20 or $50 \mu \mathrm{g} / \mathrm{mL}$ HMME. The appropriate ultrasonic irradiation time (10 s) and HMME concentration were selected from the results of these experiments. The experiment was divided into two groups that received either 20 or $50 \mu \mathrm{g} /$ mL HMME and each group was further divided into four groups: a control, ultrasound alone, HMME alone, and SDT-HMME group. After ultrasound irradiation, the cell suspensions were transferred to 96 -wells plates $(200 \mu \mathrm{L}$ cell suspension/well), followed by the immediate addition of 3-(4,5-dimethylthiazol-2-yl)-2,5-diphenyltetrazolium bromide (MTT; $5 \mathrm{mg} / \mathrm{mL}, 20$ $\mu \mathrm{L} /$ well). The cells were cultured for $4 \mathrm{~h}$ and the supernatant was aspirated after centrifugation. Following the addition of dimethyl sulfoxide (DMSO; $200 \mu \mathrm{L} /$ well) and an oscillation time of $10 \mathrm{~min}$, the optical density (OD) value of each well was measured with an automatic microplate reader set at $490 \mathrm{~nm}$. The experiment was repeated 3 times.

\section{Detection of the efficacy of HMME against hypertrophic scar fibroblasts of rabbit ear using the MTT assay}

Cells cultured as monolayer cultures and that were in the logarithmic growth phase were digested with enzymes and prepared as a single cell suspension. The cell suspensions were transferred to 96 -wells plates ( $200 \mu \mathrm{L}$ cell suspension/well) and cultured for $24 \mathrm{~h}$, after which HMME was added to the wells at a final concentration of $0,10,20,40$, and $50 \mu \mathrm{g} / \mathrm{mL}$. Three wells were allocated to each treatment group and the cells where cultured for $48 \mathrm{~h}$. MTT $(5 \mathrm{mg} / \mathrm{mL} ; 20 \mu \mathrm{L} /$ well $)$ was added before the end of the culture period. Cells were kept in culture while shaking and the OD value of each well was measured with an automatic microplate reader set at $490 \mathrm{~nm}$. The OD value of each well/group was compared with that of the control wells and calculated as the percentage of viable cells. The experiment was repeated 3 times.

\section{Assessment of the cell death model of hypertrophic scar fibroblasts of rabbit ears using Annexin-V/propidium iodide flow cytometry}

Hypertrophic scar fibroblasts of rabbit ear that were in the logarithmic growth phase were digested with commonly used enzymes, centrifuged, and subsequently prepared as a single cell suspension at $1 \times 10^{6}$ cells $/ \mathrm{mL}$. The experiments were divided into four groups that were treated with $20 \mu \mathrm{g} / \mathrm{mL}$ HMME and underwent ultrasonic irradiation of $0.50 \mathrm{~W} \mathrm{x} \mathrm{cm}{ }^{2}$ for $10 \mathrm{~s}$. After ultrasound irradiation, the cells of all four groups were transferred to $50 \mathrm{~mL}$ culture flasks for culturing. Annexin-V/propidium iodide (PI) flow cytometry was used as detection method. 


\section{Statistical analysis}

All data are reported as means \pm SD and analyzed using ANOVA followed by the Student-Newman-Keuls post-hoc test (SPSS software, version 14.0, SPSS Inc., Chicago, IL, USA). P values $<0.05$ were considered to be statistically significant.

\section{RESULTS}

\section{General observations}

After wound formation on the ventral surface of the rabbit ear, the wound was crusted and crusted off gradually. Wound healing was concluded at day 12-14 at which point the scleroma could be observed. After a tissue remodeling period of 20-22 days, the induration was more obvious, the thickness of the scar (measured with a vernier caliper) was about 2 times that of the ventral skin side of the rabbit ear, and the observed color and quality of the scar was out of the face and pale red. The extent of hyperplasia was not more than that prior to wound surgery. The scars in the model group reached their peak thickness six weeks after epithelialization of the hypertrophic scar with a thickness of about 3 times more than that of the ventral skin side of the rabbit ear (Figures 1-3).

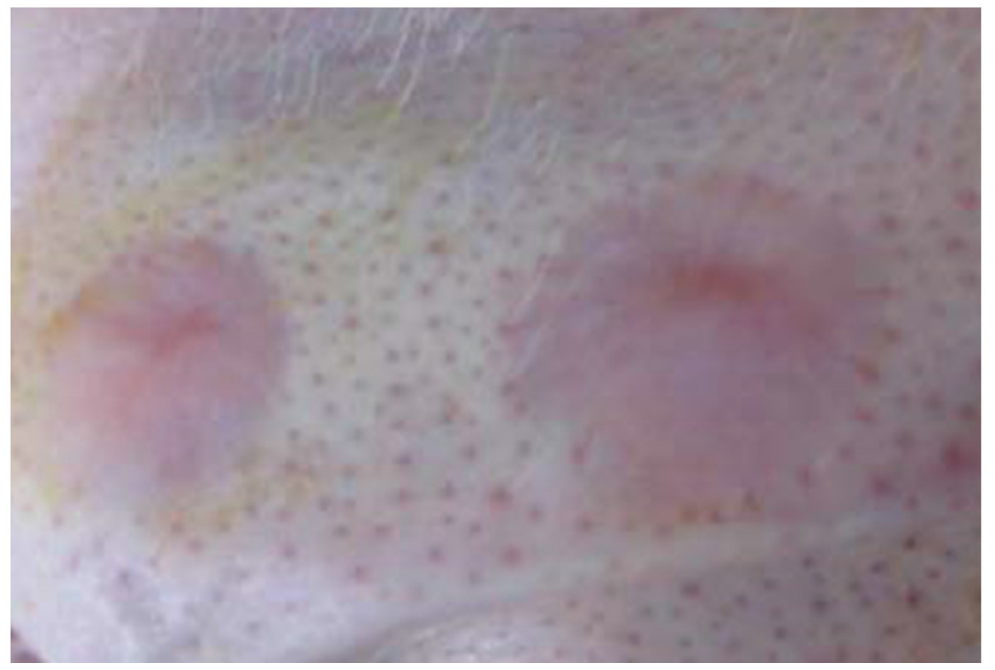

Figure 3. Scar model 6 weeks after epithelialization.

\section{Cell growth}

The hypertrophic scar fibroblast specimens were cultured for 5 days after which cell migration and adherent growth could be observed. The cells were spindle-shaped, transparent, had a full cell body, and had long flat plates, which were arranged in parallel and with a certain radian. Furthermore, round or oval nuclei were found in the center of the cells and two or three swellings were seen around the cells (Figures 4-9). 


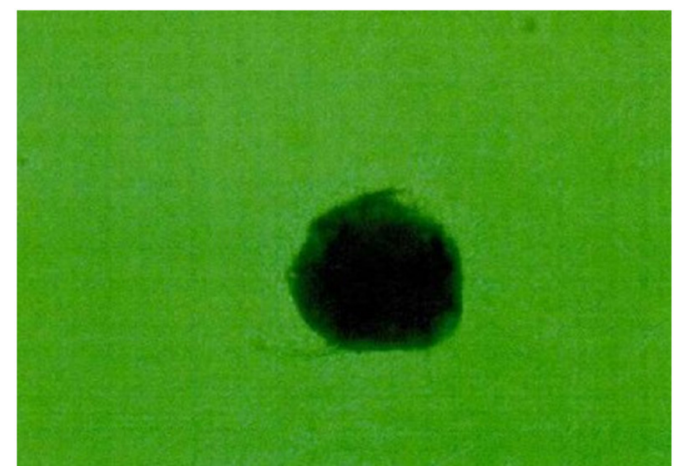

Figure 4. Crawling of the cells out of the tissue after 5 days of primary culture (100X magnification).

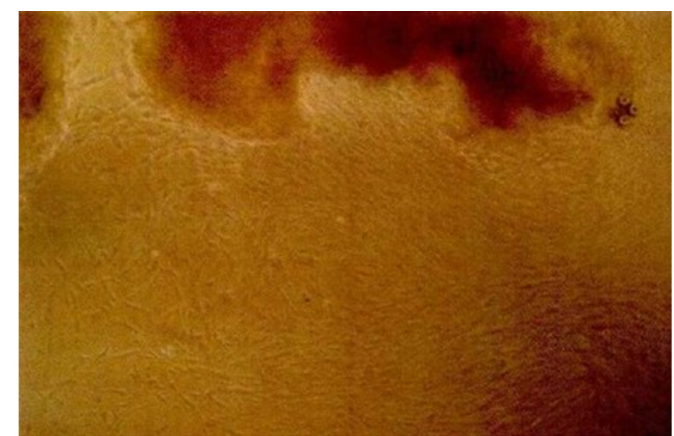

Figure 5. Crawling of the cells out of the tissue after 7 days primary culture seven days (100X magnification).

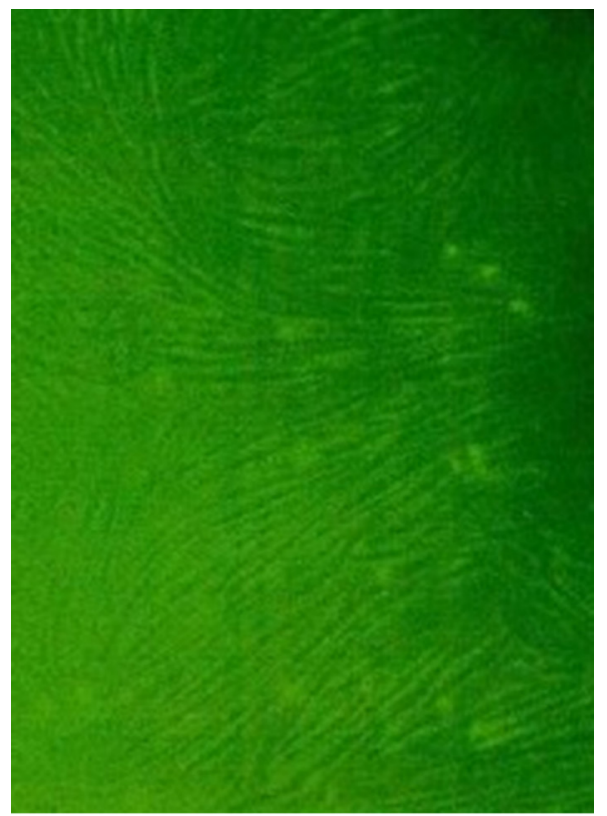

Figure 6. Cells arrayed on the bottom of the culture flask 8-10 days after primary culture (100X magnification). 


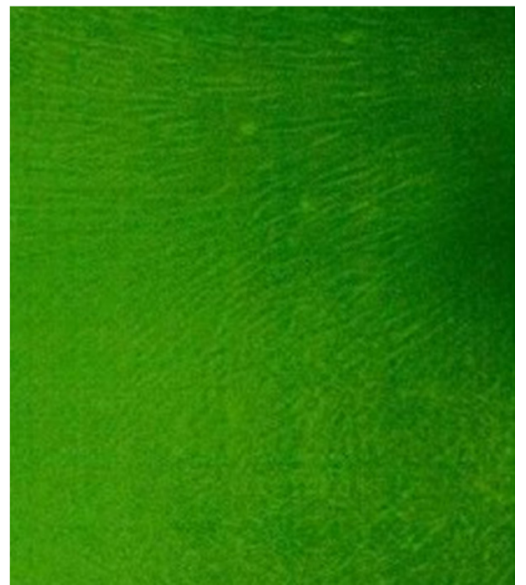

Figure 7. Cells arrayed in monolayers before generations (100X magnification).

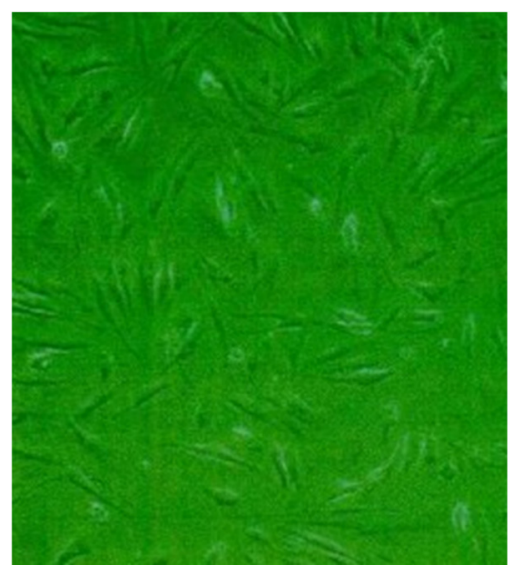

Figure 8. Healthy cell growth on the second day of subculture (100X magnification).

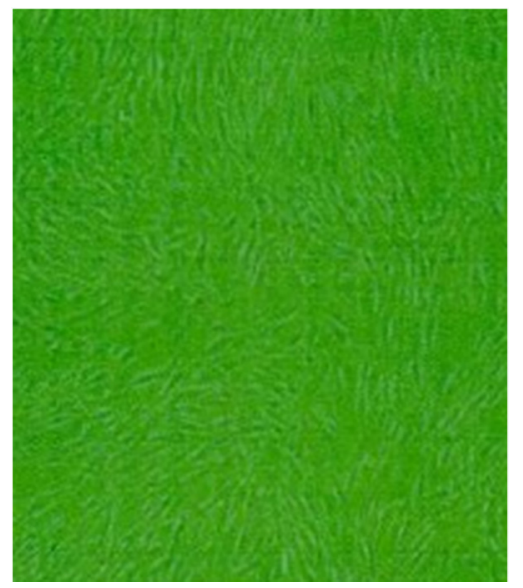

Figure 9. Cells covering the bottom of the culture flask after 7 days of subculture (100X magnification). 


\section{Effects of different ultrasonic irradiation times on the survival of hypertrophic scar fibroblasts of rabbit ears}

As shown in Table 1, the cell survival rates expressed as a percentage of the control were $95.12,74.32,58.37$, and $42.86 \%$ at an irradiation strength of $0.5 \mathrm{~W} / \mathrm{cm}^{2}$ and irradiation times of $10,30,60$, and $90 \mathrm{~s}$, respectively. The cell survival rate was significant with increasing irradiation times, which indicated a certain dose-effect relationship between the cell survival rate and the ultrasound irradiation time under these experimental conditions.

\begin{tabular}{|c|c|c|}
\hline Time (s) & Optical density value (OD) & Survival rate (\%) \\
\hline 0 & $1.7123 \pm 0.0658$ & 100 \\
\hline 10 & $1.632 \pm 0.2147$ & 95.12 \\
\hline 30 & $1.3781 \pm 0.2064$ & 74.32 \\
\hline 60 & $0.9634 \pm 0.0912$ & 58.37 \\
\hline 90 & $0.793 \pm 0.0534$ & 42.86 \\
\hline
\end{tabular}

$\mathrm{P}<0.01,0$ vs 30, 60, and $90 \mathrm{~s} ; \mathrm{P}>0.05,0$ vs $10 \mathrm{~s}$.

\section{Effects of HMME on the proliferation of hypertrophic scar fibroblasts}

No significant difference in OD values were found between the groups treated with different HMME concentrations ( $\mathrm{P}>0.05$ ), indicating that only HMME had no significant effect on the survival rate of hypertrophic scar fibroblasts (data not shown).

\section{Acute lethal effect of HMME in combination with sonodynamic therapy on hyper- trophic scar fibroblasts}

HMME-SDT had a clear lethal effect on hypertrophic scar fibroblasts; both the OD, which reflects the cell survival rate, was decreased in the HMME-SDT group compared with those of the 3 other groups. (Tables 2 and 3). The highest effect of acoustic power was observed in combination with $20 \mu \mathrm{g} / \mathrm{mL}$ HMME $(\mathrm{P}<0.05)$, which suggested that HMME had a strong acoustic sensitization effect.

Table 2. Acute lethal effect of HMME-SDT $(20 \mu \mathrm{g} / \mathrm{mL})$ on hypertrophic scar fibroblasts.

\begin{tabular}{lcc}
\hline Groups & Optical density value $(\mathrm{OD})$ & Survival rate $(\%)$ \\
\hline Control & $3.2371 \pm 0.2531$ & 100 \\
Ultrasound $(10 \mathrm{~s})$ & $2.815 \pm 0.164$ & 96.1 \\
HMME $(20 \mu \mathrm{gL})$ & $2.642 \pm 0.287$ & 94.8 \\
HMME-SDT $(20 \mu \mathrm{g} / \mathrm{mL})$ & $1.363 \pm 0.298$ & 40.1 \\
\hline
\end{tabular}

$\mathrm{P}<0.01$, the HMME-SDT $v s$ the control, ultrasound, and HMME groups. 
Table 3. Acute lethal effect of HMME-SDT $(50 \mu \mathrm{g} / \mathrm{mL})$ on hypertrophic scar fibroblasts.

\begin{tabular}{lcc}
\hline Groups & Optical density value $(\mathrm{OD})$ & Survival rate $(\%)$ \\
\hline Control & $3.1943 \pm 0.2647$ & 100 \\
Ultrasound $(10 \mathrm{~s})$ & $2.997 \pm 0.164$ & 95.1 \\
HMME $(50 \mu \mathrm{gL})$ & $2.875 \pm 0.113$ & 93.2 \\
HMME-SDT $(50 \mu \mathrm{g} / \mathrm{mL})$ & $1.687 \pm 0.2076$ & 48.4 \\
\hline
\end{tabular}

$\mathrm{P}<0.01$, the HMME-SDT $v$ s the control, ultrasound, and HMME groups.

\section{Determination of the type of cell death using Annexin V/PI flow cytometry}

The percentage of necrotic cells, early apoptotic cells, cell debris, and survival of the cells in the HMME-SDT group were $21.21 \pm 1.32,20.53 \pm 0.65,6.19 \pm 2.56$, and 50.98 $\pm 2.34 \%$, respectively, at an HMME concentration of $20 \mu \mathrm{g} / \mathrm{mL}$ as determined by flow cytometry (Table 4). These results indicated the presence of two types of cell death induced by HMME-SDT, i.e., apoptosis and necrosis (Figure 10-13).

Table 4. Effect of HMME-SDT on the type of cell death in hypertrophic scar fibroblasts.

\begin{tabular}{lc}
\hline SDT group & Percentage of cells (\%) \\
\hline Cell debris & $6.19 \pm 2.698$ \\
Early apoptosis & $23.12 \pm 1.79$ \\
Late apoptotic and necrotic cells & $22.09 \pm 2.78$ \\
Cell survival & $48.96 \pm 6.98$ \\
\hline
\end{tabular}

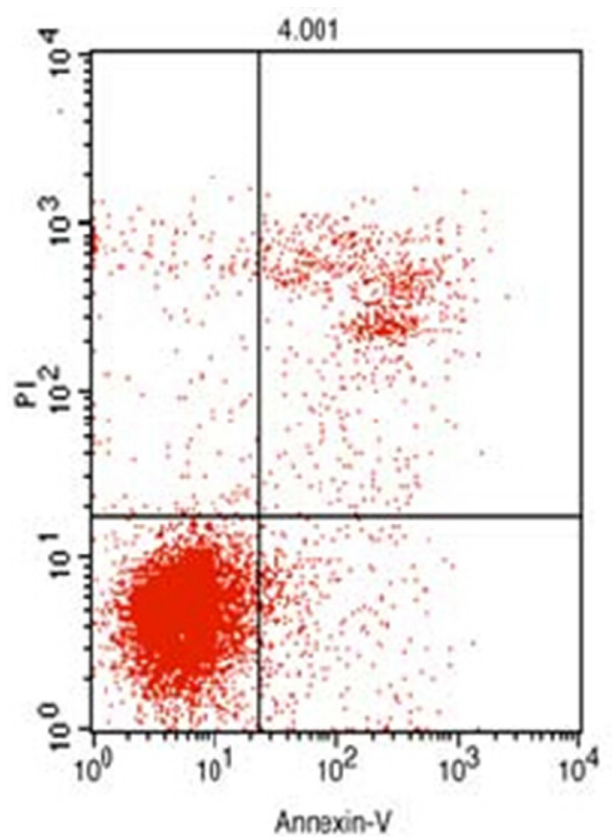

Figure 10. Type of cell death of hypertrophic scar fibroblasts treated with HMME-SDT as determined by flow cytometry after double staining with Annexin V/PI (control group). 


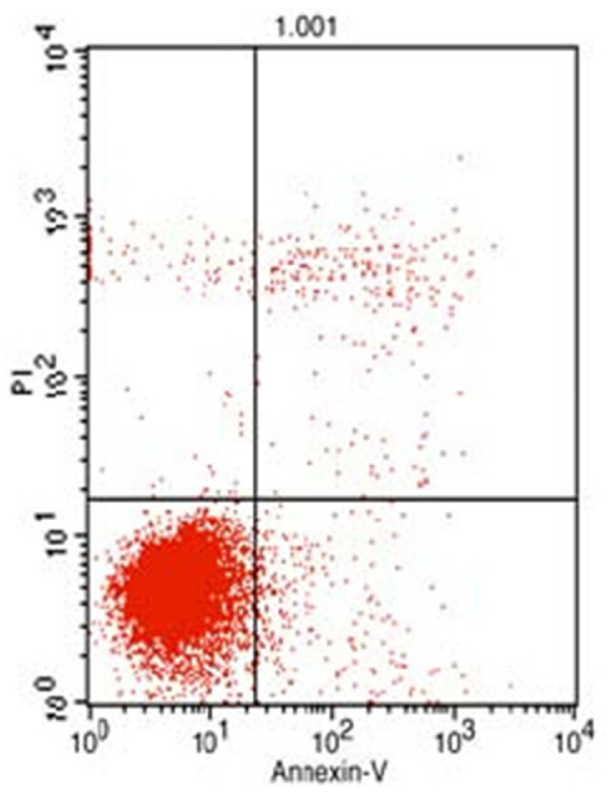

Figure 11. Type of cell death of hypertrophic scar fibroblasts treated with HMME-SDT as determined by flow cytometry after double staining with Annexin V/PI (HMME alone group).

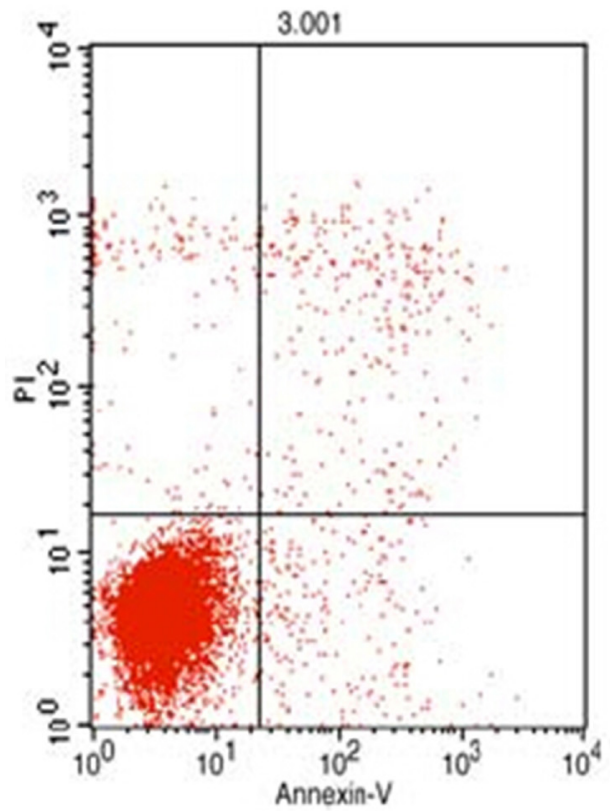

Figure 12. Type of cell death of hypertrophic scar fibroblasts treated with HMME-SDT as determined by flow cytometry after double staining with Annexin V/PI (ultrasound group). 


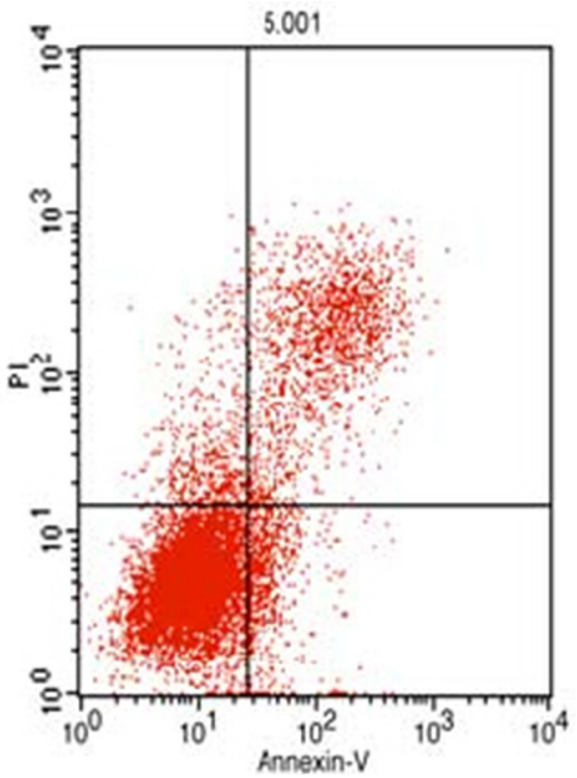

Figure 13. Type of cell death of hypertrophic scar fibroblasts treated with HMME-SDT as determined by flow cytometry after double staining with Annexin V/PI (acoustic power group).

\section{DISCUSSION}

Hypertrophic scar formation is a type of fibrous tissue hyperplasia caused by excessive wound healing after skin and tissue injury. Currently, hypertrophic scar formation and clinical research are focusing on this pathology, and clinical treatment result is not satisfactory (Liu et al., 2011). Researchers worldwide have found that SDT can induce apoptosis and inhibit cell proliferation; the underlying mechanisms and whether it is effective in the prevention of hypertrophic scar formation are, however, still unknown (Ma et al., 2011). In our previous study (Li et al., 2014) we that fibroblast proliferation may play an important role in the pathogenesis of hypertrophic scar formation. SDT is a novel therapeutic method that is increasingly used in recent years. Because it focuses the ultrasonic energy mainly on specific parts of a tumor, which activates the accumulation of hematoporphyrin in the tumor with a long retention time, thereby decreasing damage to the surrounding healthy tissue, it has broad application prospects. However, to the best of our knowledge, no research to the effects of SDT on hypertrophic scar formation has been published yet. Similarly, no reports have been published on the use of HMME as a sonosensitizer. Therefore, we applied HMME as a sonosensitizer to investigate whether its combination with SDT could have significant lethal effects on hypertrophic scar fibroblasts of the rabbit ear in vitro and whether it may provide a new approach for the treatment of hypertrophic scar formation.

We applied different concentrations of HMME on hypertrophic scar fibroblasts of rabbit ear and found that HMME did not inhibit the fibroblasts' survival. Taking into account that ultrasound treatment of tumor cells could inevitably damage the surrounding healthy cells, we performed the acoustic power experiments with an intensity of $0.5 \mathrm{~W} / \mathrm{cm}^{2}$ for $10 \mathrm{~s}$ based on our initial trial results. Similarly, according to the research using HMME photodynamic therapy, 
we used two HMME concentrations (20 and $50 \mu \mathrm{g} / \mathrm{mL}$ ) to investigate potential differences in the effect of SDT on hypertrophic scar fibroblasts and found that the survival rate of hypertrophic scar fibroblasts was significantly decreased in the HMME acoustic power group. In contrast, that of the hypertrophic scar fibroblasts in the HMME alone group was not changed. The fibroblasts' survival rate in the HMME alone group was, however, lower than that of those in the ultrasound alone group, which indicated that a certain level of ultrasound could activate the effects of HMME on the hypertrophic scar fibroblasts. Therefore, it may be suggested that HMME might be an effective sonosensitizer .

Our study found that the lethal effect of $20 \mu \mathrm{g} / \mathrm{mLHMME-SDT}$ on hypertrophic scar fibroblasts was higher than that of $50 \mu \mathrm{g} / \mathrm{mL}$ HMME-SDT (cell survival rates of 40.1 and $48.4 \%$, respectively), which may be related to a concentration saturation effect of HMME at these concentrations. Flow cytometry analysis showed that cell death due to both apoptosis and necrosis was present in the HMME acoustic power group.

In summary, our results showed that HMME could be an effective sonosensitizer as HMME-SDT had an acute toxic effect on hypertrophic scar fibroblasts, causing necrosis, apoptosis, and a decreased cell survival rate.

\section{ACKNOWLEDGMENTS}

Research supported by grants from the Health Department of Heilongjiang Province (\#2012-690, \#2009-174), the Project of Science and Technology Program of Heilongjiang Province (\#GC10C303-4), the Science and Technology Talents Program of Harbin (\#2012RFXXS066, \#2014RFXGJ041, \#2014RFQGJ094, \#2014RFXGJ035), and the Department of Education Project of Heilongjiang Province (\#11541147).

\section{REFERENCES}

Dai SC, Hu SS and Zhan Q (2011). Sonodynamic therapy with hematoporphyrin monomethyl ether induced apoptosis of C6 glioma cells in vitro. J. Exp. Surg. 28: 727-729.

Li W, Fei JF, Li XF, Qu ZW, et al. (2014). Effects of HMME-STD on hypertrophic scar of rabbit ear. Chin. J. Med. Aesth Cosmet. 20: 58-62.

Liu Y, Cen Y, Chen JJ, Li BL, et al. (2011). The extracellular signal regulated kinase and stress activated protein kinase pathway protein in the expression of fibroblasts in keloid. Zhonghua Wai Ke Za Zhi [Chin. J. Surg.]. 25: 1367-1370.

Lv Y, Fang M, Zheng J, Yang B, et al. (2012). Low-intensity ultrasound combined with 5-aminolevulinic acid administration in the treatment of human tongue squamous carcinoma. Cell. Physiol. Biochem. 30: 321-333.

Ma J, Li XJ, Liu L, Zhou CL, et al. (2011). Inhibiting the expression of Survivin gene induced fibroblast apoptosis of keloid with small interfering RNA. J. Exp. Surg. 28: 923-925.

Morris DE, Wu L, Zhao LL, Bolton L, et al. (1997). Acute and chronic animal models for excessive dermal scarring: quantitative studies. Plast. Reconstr. Surg. 100: 674-681. 